\title{
La intervención en el territorio como una herramienta para la resiliencia ante el cambio climático
}

\section{Intervention in the territory as a tool for resilience to climate change}

\author{
Marinero-Orantes, Edgar Antonio; Vargas-Cañas, José Isidro; Molina, \\ Adalila; Zuniga-González, Carlos Alberto; Sól-Sánchez, Angel; Editor \\ Academico Dr. Pedro Jose Toruño
}

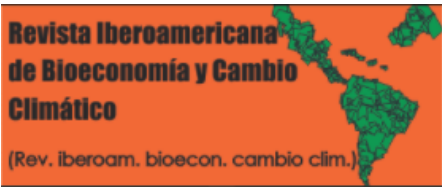

Edgar Antonio Marinero-Orantes

edgar.marinero@ues.edu.sv

Universidad de El Salvador, El Salvador

José Isidro Vargas-Cañas

Universidad de El Salvador,, El Salvador

iD Adalila Molina

lilam7@yahoo.com

Universidad Nacional Autónoma de Nicaragua,

UNAN León, Nicaragua

Carlos Alberto Zuniga-González

czuniga@ct.unanleon.eduni

Universidad Nacional Autónoma de Nicaragua, León.,

Nicaragua

(iD) Angel Sól-Sánchez

sol@colpos.mx

Colegio de Postgraduados Campus Tabasco, México,

Mexico

Editor Academico Dr. Pedro Jose Toruño

Universidad Nacional Autonoma de Nicaragua Leon.

Nicaragua, Nicaragua

Revista Iberoamericana de Bioeconomía y Cambio

Climático

Universidad Nacional Autónoma de Nicaragua, León, Nicaragua ISSN-e: 2410-7980

Periodicidad: Semestral

vol. 2, núm. 4, 2016

czuniga@ct.unanleon.edu.ni

Recepción: 28 Febrero 2016

Aprobación: 15 Diciembre 2016

URL: http://portal.amelica.org/ameli/journal/394/3941751004/

DOI: https://doi.org/10.5377/ribcc.v2i4.5928

Autor de correspondencia: edgar.marinero@ues.edu.sv
Resumen: El presente documento describe la implementación de acciones en cooperación con Universidades Centroamericanas en relación con la Universidad de Alcalá, España, específicamente con lo relacionado con las actividades implementadas en el marco del PROGRAMA UNIVERSITARIO DE DESARROLLO LOCAL con la finalidad de establecer la vinculación con el territorio. Además se presenta esta metodología como un aporte de la academia ante los impactos del cambio climático, pues es de vital importancia el aporte de las escuelas de educación superior en cuanto a la innovación para promover la resiliencia como estrategia para disminuir el impacto del cambio climático. Se plantea algunas conclusiones y los pasos seguidos para impactar los territorios.

Palabras clave: territorio, resiliencia, academia, cambio climático.

Abstract: This document describes the implementation of actions in cooperation with Central American Universities in relation to the University of Alcalá, Spain, specifically related to the activities implemented in the framework of the UNIVERSITY DEVELOPMENT PROGRAM LOCAL with the purpose of establishing the link with the territory . In addition, this methodology is presented as a contribution of the academy to the impacts of climate change, as it is of vital importance the contribution of higher education schools in terms of innovation to promote resilience as a strategy to reduce the impact of climate change. Some conclusions and steps are taken to impact the territories.

Keywords: territory, resilience, academia, climate change. 


\section{ANTECEDENTES}

La Facultad Multidisplinaria Paracentral (FMP), es una de las doce facultades que actualmente integran la Universidad de El Salvador, con sede en la ciudad de San Vicente, departamento de San Vicente, El Salvador, fue creada en el año de 1989 y desde entonces su proyecto académico se ha enmarcado dentro de su relación interinstitucional para ejecutar acciones de intervención en el territorio que le permitan fortalecer sus tres funciones básicas como son la docencia, la investigación y la proyección social, por lo que en los últimos años se vio en la necesidad de insertarse a nivel regional para el diseño de programas de carácter académico como es el caso del Programa Universitario de Desarrollo Local, esto propició la participación en la red Interuniversitaria de Cooperación para el Desarrollo de Centroamérica, por lo que bajo cooperación se ha desarrollado acciones relacionas de cooperación con los actores sociales de la zona de influencia institucional; propiciando la visión de que las universidades no somos las responsables del desarrollo local, pero somos catalizadores de los procesos de desarrollo en el territorio con aportes a la formación de capacidades.

En el marco de la cooperación varias Universidades Centroamericanas en relación con la Universidad de Alcalá, España, han venido analizando la definición del PROGRAMA UNIVERSITARIO DE DESARROLLO LOCAL con la finalidad de establecer la vinculación con el territorio, y que al mismo tiempo se desarrolló en la UNAN, León, Nicaragua.

Siendo la Facultad reconocida en su entorno social como la única institución pública de educación superior, tiene grandes posibilidades de actuar sobre el territorio de la Zona Paracentral de El Salvador, impulsando el Desarrollo territorial, con lo cual genera conocimientos útiles y aplicables sobre los recursos naturales. Esto permitió acompañar con acciones de investigación y proyección social, a los gobiernos locales, ONG's, entidades que se encuentran en el territorio, prestando servicios técnicos y científicos y ayudando a desentrañar problemas.

\section{Algunos aspectos RELACIONADOS CON LAS UNIVERSIDADES EN EL NUEVO POSICIONAMIENTO REGIONAL Y COMO ENTES PROMOTORES DEL DESARROLLO TERRITORIAL.}

La evolución regional ha marcado un nuevo rol a las Universidades en el marco del desarrollo centroamericano, especialmente en el siglo XXI donde las inducen a modelos más flexibles de educación, ya que el desarrollo tendrá su fundamento en la intensificación de la tecnología y el conocimiento, por ello se incluyen como ejes claves: la diversificación de la oferta educativa, la vinculación de la Universidad con la sociedad, que marca el avance hacia la consolidación de una institucionalidad universitaria articulada en redes y vinculada a su entorno.

Es de destacar que en octubre de 1993, los Presidentes de los estados de América Central suscribieron el Protocolo al Tratado General de Integración Económica Centroamericana, conocido como "Protocolo de Guatemala". Mediante este instrumento se plantea el compromiso de procurar alcanzar la libre movilidad de los factores productivos en la región. Para ello en su artículo 31 establecen que: "Los estados parte acuerdan armonizar sus legislaciones para el libre ejercicio de las profesiones universitarias en cualquier país de la región, a efecto de hacer efectiva la aplicación del Convenio sobre el Ejercicio de Profesiones Universitarias y Reconocimiento de estudios universitarios, suscrito el 22 de junio de 1962, en la ciudad de San Salvador, Republica de El Salvador, el cual es de aplicación plena en los Estados Contratantes de ese Conveio".

Notas DE AUTOR

edgar.marinero@ues.edu.sv 
Lo anterior, pone de manifiesto, que tanto el SICA como el CSUCA han articulado acciones para lograr la integración a nivel de la educación superior, y una de las formas que ha desarrollado mayores réditos en este sentido, son las redes de Universidades Centroamericanas que articuladas con Universidades de otras latitudes han permitido implementar programas, proyectos e iniciativas en los campos de las funciones sustantivas de las Universidades (Arias y Molina, 2006; Vargas, Sierra y del Romero, 2006; Boeren, 2003).

\subsection{El territorio como nuevo concepto de desarrollo y como base de la planificación para la integración regional}

Los enfoques territoriales para promover el desarrollo marcan una tendencia a consolidarse como planteamiento alternativo ante los múltiples fracasos suscitados de las diversas intervenciones que no lograron reducir la pobreza, agudizaron la fragilidad de los ecosistemas y aumentando la vulnerabilidad de los territorios.

Para abordar el marco de referencia es necesario partir de siete enfoques-teorías consideradas claves para sustentar el desarrollo: (i) multidimensión de la pobreza, (ii) territorialidad, (iii) desarrollo económico local, (iv) capital social, (v) eficacia de la ayuda, (vi) competencia sistémica, y (vii) fortalecer la resiliencia, los cuales de forma articulada dan base a un enfoque que permita avanzar hacia el planteamiento del territorio y se resume a seguir:

"El concepto de territorio después de haber quedado postergado durante las pasadas décadas por el empuje de un desarrollismo urbanizador... regresa cargado de nuevos significados, en parte recibidos de lo que siempre se ha conocido como la naturaleza y sus recursos, pero también enriquecido con los elementos construidos, el legado cultural e histórico" (Sal, 2000)

Pero para lograr encontrar una sinergia entre el desarrollo regional centroamericano y el enfoque territorial planteado se debe en primera instancia aceptar, que el enfoque de la pobreza es un concepto que se ha construido sobre la base de la evolución desde una perspectiva que consideraba las carencias o necesidades básicas insatisfechas hasta la incorporación de seis fuentes de bienestar para personas y hogares: Ingresos, servicios, propiedad, habilidades y destrezas para la vida y el trabajo, tiempo disponible para la realización en distintas esferas y autonomía de las personas (CEPAL, 2004). En ese sentido, es claro que las sociedades centroamericanas no solamente tienen población que en grandes proporciones tienen privadas sus necesidades, sino que además carecen de tiempo y posibilidades para buscar opciones para satisfacerlas y generar ingresos propios, lo cual plantea la necesidad de analizar la relevancia en el uso del tiempo y el acceso a activos (Moreno, 2002; del Romero y Vargas, 2005).

El enfoque territorial enfrenta actualmente un contexto regional donde prevalecen nuevas organizaciones, acentuadas migraciones y desigualdad creciente entre otras. Es decir que, se habla de un enfoque centrado en el territorio considerando cuatro aspectos centrales que son: el desarrollo económico local, la gestión y manejo de los recursos naturales, el desarrollo del rescate de los marcos culturales que se transforman en activos intangibles y la promoción del capital humano y social (Alonso y Ocampo, 2011).

Este tipo de enfoque surge a partir de la creciente comprensión sobre la necesidad de colocar a los pobres y a todos los aspectos de su realidad y sus estilos de vida al centro del desarrollo, a su vez, manteniendo la sostenibilidad de los recursos naturales para generaciones presentes y futuras.

Los enfoques de desarrollo económico local han estado sustentados en dos corrientes de políticas públicas ampliamente abordadas en documentos de la cooperación internacional, uno de ellos es la descentralización y otro la municipalización. En ambos casos el tema central ha sido la forma que debe acoger este enfoque en los territorios para motivar a los actores a participar. En Centro América se han adoptado modalidades diversas, destacando las Agencias de Desarrollo Económico Local que existen en toda la región pero principalmente en El Salvador, Honduras y Guatemala, habiendo sido modelo para experiencias exitosas en Ecuador y Republica 
Dominicana. Pero al mismo tiempo, se ha avanzado en liderazgo territorial de actores en la esfera económica tal es el caso de fundaciones, organizaciones de productores y otras que han logrado posicionamiento en el tema del desarrollo económico local ya sea por la vía del valor agregado, o por la del encadenamiento productivo. (Para mayor detalle referirse a Diamiani (2008), Albuquerque (2004) y otros).

En síntesis es un enfoque integral centrado en la educación superior los elementos centrales de la multidimención de la pobreza, territorialidad, acceso a activos, capital social, desarrollo económico local y eficacia de la ayuda. Paralelamente, algunas experiencias sin citar el enfoque de competitividad sistémica que busca generar un análisis a distintos niveles, ha logrado intervenir de manera articulada en los territorios vía la vinculación Universidad Sociedad con resultados que aportan a un análisis centrado en este tipo de competitividad. Y aportando a la resiliencia en los territorios donde las amenazas naturales impactan la cotidianidad de los asentamientos humanos, generando niveles de recuperación de los daños y la generación de medidas para la adaptación al cambio del clima, tal como lo define Orozco y Guevara (2011) donde señalan que la resiliencia es el aumento de la capacidad de los sectores, territorios y sistemas sociales y ambientales expuestos al Riesgo de Desastres, para resistir al impacto y alcanzar un nivel aceptable en su funcionamiento y su estructura, mediante el ajuste y la auto-organización. Esto permite lograr una mejor protección futura y mejorar las medidas de reducción de riesgo de desastres asociados al Cambio Climático. Idealmente, el objetivo de gestión del riesgo de Cambio Climático es pasar de la vulnerabilidad a la resiliencia.

\section{LA RESILIENCIA}

Uno de los aspectos fundamentales para lograr despertar las capacidades de los territorios en cuanto recuperar su funcionamiento luego del impacto del cambio climático, se enmarca en el nuevo concepto de resiliencia. Y para lograrlo es fundamental consolidar una organización que sea consciente y solidaria con las familias que habitan en esas zonas más vulnerables. Además es parte importante la conciencia de las capacidades de los territorios en cuanto a los recursos existentes para lograr la recuperación de los asentamientos humanos.

El aumento de la resiliencia es el aumento de la capacidad de los sectores, territorios y sistemas sociales y ambientales expuestos al Riesgo de Desastres, para resistir al impacto y alcanzar un nivel aceptable en su funcionamiento y su estructura, mediante el ajuste y la auto-organización. Esto permite lograr una mejor protección futura y mejorar las medidas de reducción de riesgo de desastres asociados al Cambio Climático.

Idealmente, el objetivo de gestión del riesgo de Cambio Climático es pasar de la vulnerabilidad a la resiliencia. Considerando que el impacto del Cambio Climático es inevitable y que las tendencias globales muestran un incremento a las pérdidas asociadas a riesgos climáticos, el aumento de la resiliencia es un requisito esencial para fortalecer y complementar las alternativas de adaptación al Cambio Climático. Aunque la disminución de las emisiones de gases de efecto invernadero es fundamental para reducir los riesgos de desastre en el futuro (mitigación), una tarea inmediata para fortalecer la resiliencia del país consiste en la preparación para condiciones meteorológicas más extremas mediante programas de reducción de los riesgos de desastre (por ejemplo, programas de educación pública sobre los riesgos, sistemas de alerta temprana y preparación para casos de desastre. (Orozco y Guevara, 2011).

El Marco de Acción de Hyogo 2005-2015, donde se aborda con especial énfasis la resiliencia de las naciones las comunidades ante los desastres, incluye un plan para reducir la vulnerabilidad a fenómenos atmosféricos intensos y al Cambio Climático, y para la adaptación a la amenaza de aumentos futuros de los extremos climáticos. De acuerdo con éste, la resiliencia permitirá a un Territorio y a las Comunidades que lo ocupan entender a largo plazo sus procesos de crecimiento y mejoramiento de competitividad, al mismo tiempo adaptarse a fenómenos alarmantes y cambios complejos. La incorporación del concepto de resiliencia en la política y la práctica será una tarea importante para todo el país en el futuro que lo hará mucho más resistente ante los cambios (de temperaturas, de nivel de precipitaciones o de cualquier otra índole). (Orozco y Guevara, 2011). 


\section{UNA ACCIÓN ARRAIGADA EN EL TERRITORIO}

La filosofía de la intervención territorial, se sustenta en la generación de "capacidades compartidas" entre las universidades para su fortalecimiento institucional y, de ese modo, enfrentar juntas los problemas de desarrollo de la región, "especialmente los de los sectores más débiles y vulnerables".

Asimismo, la creación de redes de área o proyecto está generando capacidades de investigación, organización, docencia y mayor pertinencia ante los problemas concretos (Vargas y del Romero, 2008; Sumpsi, 2006).

La vinculación de una universidad, se establece cuando alguno de sus profesores o técnicos se suma, con el apoyo institucional de su universidad, a un proyecto con los actores locales de los territorios del país. De este modo, el compromiso y la voluntad personal y el institucional se conjugan para que un proyecto tenga impacto en el territorio.

La universidad está estructurada, por sus autoridades académicas y por los estudiantes que operativizan los acuerdos, y los proyectos. El organismo institucional tiene como objetivo principal valorar los proyectos, analizar otros posibles proyectos y apoyar a los grupos que intervienen el territorio.

La FMP en relación al Programa Universitario de Desarrollo Local ha ejecutado acciones en el territorio con impacto en las comunidades y que aporten a la capacidad de los sectores para adaptarse y recuperar las capacidades para disminuir los impactos del cambio climático.

\section{LAS LÍNEAS ESTRATÉGICAS}

Este modelo se basó en considerar a todos los sectores, instituciones y actores locales, nacionales e internacionales, con el fin de articular acciones y esfuerzos que impacten en la zona de influencia institucional; y que sean flexibles permitiendo el aglutinamiento los esfuerzos del personal académico, estudiantes, funcionarios, líderes comunitarios y tomadores de decisión.

La implementación del Programa Universitario de Desarrollo Local Sostenible, exigió primeramente su institucionalización y la conformación de un equipo de trabajo para organizar actividades e incorporar acciones en el Plan Operativo Anual Institucional.

En respuestas a las necesidades del territorio la Facultad desarrolló las siguientes estrategias:

FORMACION DE TALENTOS HUMANOS, en respuesta a las necesidades y demandas del territorio.

INTEGRACION DE ESTUDIANTES EN PROYECCION SOCIAL, en respuesta a la demanda de servicios en comunidades de poco acceso y la oportunidad de aprender-haciendo en la realidad del territorio.

INVESTIGACION, para la generación de conocimientos y tecnologías en respuesta a las demandas del territorio

ARTICULACION DE LA GESTIÓN: Programa de Desarrollo Local Sostenible en el gobierno local y territorial con la definición de: acuerdos, convenios, alianzas, cartas de entendimiento.

PLANIFICACION, SEGUIMIENTO (SISTEMATIZACION) Y EVALUACION, con la participación de la sociedad.

Por tanto, a partir de éstas estrategias se pudo obtener una serie de insumos esenciales para la formulación y caracterización de un proceso que garantizó la intervención territorial, el cual promovió el conocimiento del riesgo de desastres, permitiendo la toma de decisiones asertivas y orientadas para desarrollo de procesos dinámicos y asequibles para la resiliencia. 


\section{Conclusiones}

- Se inició la consolidación de un método que encamina a la universidad a una solidaridad crítica de los problemas. La organización y participación, es clave para la consolidación y adopción de un proyecto en fase de ejecución.

- El abordaje de problemas comunes es condición indispensable para que los proyectos y programas sean sostenibles y fortalezcan la resiliencia en los territorios.

- La formación de equipos es una condición necesaria para la sostenibilidad y los buenos resultados de proyectos y programas. La existencia de equipos cohesionados y con funciones y responsabilidades compartidas permite abordar en mejores condiciones los proyectos.

- La formulación y gestión de proyectos plurianuales regionales es un modo de orientar su realización para alcanzar resultados e, incluso, poder ver algunos impactos.

\section{Referencias Bibliograficas}

Alburquerque Llorens, F. (2004). Desarrollo económico local y descentralización en América Latina. Revista de la CEPAL. 82, 157-170

Alonso, J. A., y Ocampo, J. A. (Eds.). (2011). Cooperación para el desarrollo en tiempos de crisis. Fondo de cultura económica.

Arias, S. y Molina, E. (2006). Universidad y Cooperación al Desarrollo. UAM: Cuadernos Solidarios.

Boeren. A. (2003). "Soplando al viento: tiempos de cambio para la cooperación al desarrollo en educación superior". Disponible en: www.cecod.net/cooperacion_universitaria.htm.

Vargas, M., Sierra, F. C., y del Romero Guerrero, A. (2006). Fortalecimiento de sistemas universitarios del sur: claves y retos del hermanamiento entre UNAN-León de Nicaragua y la Universidad de Alcalá. In Ponencias y comunicaciones (pp. 527-553). Universidad Complutense de Madrid.

Diamani, O. (2008). Desarrollo rural desde una perspectiva territorial: Estudio comparativo de casos en Asia y América Latina. Santiago: Rimisp-Centro Latinoamericano para el Desarrollo Rural.

del Romero Guerrero, A., \& Vargas, M. (2005). Se hace camino al andar, o, Aprendiendo a cooperar entre universidades del Sur y del Norte. Servicio de Publicaciones, Universidad de Alcalá.

Moreno, E. (2002). Evaluation Report of the Collaboration between Sweden and the Public Universities of Nicaragua. Agencia Sueca de Cooperación Internacional para el Desarrollo (ASDI/SAREC)

Orozco, R., \& Guevara, O. (2011). Gestión Integrada del Riesgo de Desastres: Pautas metodológicas para la formación y administración de proyectos en Gestión del Riesgo. Medellín, Colombia: Escuela Latinoamericana de Cooperación y Desarrollo.

Sumpsi, J. M. (2006). Relaciones entre las transformaciones institucionales y productivas en el marco del desarrollo territorial rural. Santiago de Chile: RIMISP-IDRC.

Sal, A. G. (2000). Territorio henares: cultura y naturaleza en un espacio compartido. Servicio de Publicaciones. Universidad de Alcalá.

Vargas, M., y Del Romero, A. (2008). Entre la oportunidad y la necesidad: Génesis y retos de una nueva Red Interuniversitaria de Cooperación para el Desarrollo de Centroamérica (RED GIRA). In UAB: Actas del IV Congreso Universidad y Cooperación. y administración de proyectos en Gestión del Riesgo. Medallin, Colombia (Vol. 115).

CEPAL (2004). Panorama Social de América Latina. Santiago de Chile. Cuadernos de la Cepal, (92). 